website extra

Additional information appears on the Mental Health website wealth website www.ebmentalhealth. com/supplemental

\title{
Cognitive training may improve targeted cognitive functions in older adults
}

Ball $K$, Berch D, Helmers $K$ et al. Effects of cognitive training interventions with older adults. A randomized controlled trial.JAMA 2002 Nov;288:2271-81.

QUESTION: Do interventions targeting memory, reasoning and cognitive speed affect the mental abilities and everyday activities of older adults?

Design

Randomised controlled trial. Analysts were blind to treatment allocation.

\section{Setting}

6 cities in the United States; March 1998-December 2001.

\section{Participants}

2802 older adults recruited from senior housing, community centres, clinics and driver's licence registries. Mean age 73.6 years (range $65-94$ ); $76 \%$ women; $73 \%$ white. Exclusion criteria were age under 65 years; not living independently; recent cognitive training; substantial cognitive decline; Alzheimer's disease; medical conditions with increased risk of functional decline or death, or severe loss of vision, hearing or communicative ability.

\section{Intervention}

Participants received 10 group training sessions in either (1) memory (verbal episodic memory); (2) reasoning (ability to solve problems following a serial pattern); (3) speed of processing (visual search and identification), or were allocated to a non-contact control group. 11 months later, 4 booster sessions were offered to a random sample of $60 \%$ of participants. Follow up data were collected from $80 \%$ after 2 years.

\section{Main outcome measures}

Cognitive function and cognitively demanding everyday function were measured using composite scores on a variety of tests and the Activities of Daily Living scales.

Source of funding: National Institute of Aging; National Institute of Nursing Research.

For correspondence: $K$ Ball, Center for Research on Applied Gerontology, University of Alabama at Birmingham, Alabama. kball@uab.edu

\section{Main results}

All 3 interventions improved the targeted cognitive ability (memory, reasoning or speed of processing) compared to baseline. Improvements were noted immediately after the intervention and at 2-year follow up. Booster training increased gains in speed and reasoning. At 2 years, the authors did not detect any effects on everyday functioning, likely due to the lack of functional impairment at baseline.

\section{Conclusions}

Poor cognitive function is associated with reduced independent living in older adults. This study suggests that 10 sessions of cognitive training may improve targeted cognitive abilities, but there were no benefits on everyday cognitive functioning after 2 years.

\section{COMMENTARY}

Ball et al report effect sizes ranging from small to large. Following the intervention, $26 \%$ of the memory group, $74 \%$ of the reasoning group and $87 \%$ of the speed training group had significant improvements in memory, reasoning and speed of processing, respectively. Apart from everyday cognitive functioning, these improvements were largely maintained 2 years later. It is interesting to note that while most older adults report declines in memory, the memory intervention had the smallest effect.

This study addresses a number of limitations of previous research, including small sample sizes, minimal follow-up periods and lack of adequate control groups. The intervention conditions were used as training exposure and social contact control conditions for each of the other interventions.

The impact of the interventions appears to be limited to the particular cognitive domain targeted and does not generalise to everyday functioning. The authors suggest that ceiling effects for measures of functioning may explain this finding. This is unlikely because all participants were cognitively intact, yet they demonstrated improvements in cognitive functioning. Adherence is another possible explanation for the lack of effect on everyday activities. We know that participants mastered the skills because their cognitive function improved, but we do not know if participants used these techniques in their daily lives. For example, did participants apply the mnemonic techniques they learned to help them remember the people's names? The outcome measures used may also have affected findings about everyday activities. The tests of everyday functioning were not specific to the cognitive skills that participants were taught. For example, driving habits were included as an outcome, yet this relates little to the skills taught in the intervention. Similarly, tests of everyday speed were largely dependent upon physical speed, yet the intervention focused on improving mental speed. Cognitive interventions need to teach people how to apply the targeted skills to specific everyday life activities. Assistant Professor Gretchen A Brenes, $\mathrm{PhD}$ Wake Forest University School of Medicine North Carolina, USA 